\title{
IMPACTO DA VACINAÇÃO DA COVID-19 NOS PROFISSIONAIS DA SAÚDE EM MUNICÍPIOS DO RS - BRASIL
}

\author{
Júlia Vaz Schultz'; Mariana Zancan Tonel ${ }^{2}$; André Flores dos Santos ${ }^{3}$; Alencar \\ Kolinski Machado4; Ivana Zanella ${ }^{5}$; Solange Binotto Fagan ${ }^{6}$; Mirkos Ortiz \\ Martins $^{7}$
}

\section{RESUMO}

Este trabalho analisa o impacto da vacinação para COVID-19 em novos casos da doença, a partir da imunização do grupo prioritário de profissionais da saúde nos municípios mais populosos do RS: Porto Alegre, Caxias do Sul, Canoas, Pelotas e Santa Maria. Os resultados mostram, via modelos de regressão matemática, o comportamento para novos casos de COVID-19 a partir de três cenários: i) grupo sem vacinação, ii) grupo imunizado e iii) média semanal acumulada. Observa-se uma redução significativa do número de casos confirmados da COVID-19 com o avanço da imunização dos profissionais de saúde nas cidades analisadas.

Palavras-chave: Infecção por coronavírus, vacinação, grupos prioritários, modelagem

\footnotetext{
${ }^{1}$ Aluna do Programa de pós-graduação em Nanociências - Universidade Franciscana. E-mail: juliavschultz@gmail.com

${ }^{2}$ Bolsista de pós-doutorado na Universidade Franciscana; marianaztonel@gmail.com

${ }^{3}$ Aluno do Programa de pós-graduação em nanociências - Universidade Franciscana. E-mail: andre flores2009@hotmail.com

${ }^{4}$ Professor do Programa de pós-graduação em Nanociências- Universidade Franciscana. E-mail: alencarkolinski@gmail.com

${ }^{5}$ Professora do Programa de pós-graduação em Nanociências- Universidade Franciscana. E-mail: ivanazanella@gmail.com

${ }^{6}$ Coordenadora do projeto. Professora do Programa de pós-graduação em Nanociências- Universidade Franciscana. E-mail: solange.fagan@gmail.com

${ }^{7}$ Coordenador do projeto. Professor do Programa de pós-graduação em Nanociências- Universidade Franciscana. E-mail: mirkos@gmail.com
} 
Eixo Temático: Tecnologia, Inovação e Desenvolvimento Sustentável (TIDS).

\section{INTRODUÇÃO}

A humanidade enfrenta uma das maiores crises sanitárias da contemporaneidade ao encarar a doença causada pelo novo coronavírus (SaRS-CoV-2) denominada COVID-19 (do inglês coronavírus disease 2019). O SaRS-CoV-2 surgiu no final de 2019 em Wuhan - China, causando um grave problema de saúde pública e uma crise econômica e social sem precedentes(LI et al., 2020; WU et al., 2020; ZHOU et al., 2020). Até o momento, as medidas de contenção da doença estão relacionadas, principalmente, com a prevenção como o uso de máscara(GARCIA, 2020), distanciamento social, lavagem das mãos, assim como o desenvolvimento de novos tratamentos e vacinas ("4a edição COVID-19Guia Orientador para o enfrentamento da pandemia na Rede de Atenção à Saúde", [s.d.]; GARCIA; DUARTE, 2020).

Do ponto de vista epidemiológico, as vacinas são consideradas uma tecnologia de excelente custo-benefício e alto grau de resolutividade para a saúde pública. As evidências científicas demonstram que a eliminação e/ou controle das doenças transmissíveis está intrinsecamente ligada ao conceito de imunidade coletiva(CARACILO CARVALHO BIVAR et al., 2021). O Brasil é considerado um dos países com o maior programa de vacinação pública do mundo, com campanhas regulares para grupos específicos. A vacinação é uma importante aliada na redução de casos e mortes por causa da COVID-19. Até o momento três vacinas estão autorizadas no Brasil para uso emergencial pela Anvisa: Corona Vac/Instituto Butantan, Janssen e Covishield (Serum Institute of India, em parceria com a AstraZeneca/Universidade de Oxford/Fiocruz), duas com uso definitivo: AstraZeneca/Fiocruz e Pfizer/Biontech, e outras duas autorizadas para importação excepcional: Covaxin e Sputnik("4a edição COVID-19- Guia Orientador para o enfrentamento da pandemia na Rede de Atenção à Saúde", [s.d.]). 
Nesse sentido, o marco inicial da vacinação contra a COVID-19 no Brasil foi o dia 17 de janeiro de 2021, já no estado do RS foi a partir de 18 de janeiro de 2021 com a vacina Corona Vac/Instituto Butantan. A partir do dia 24 de janeiro, foi recebida no RS a primeira remessa de vacina Oxford/AstraZeneca. Os profissionais da saúde foram um dos primeiros grupos prioritários a receberem a vacina(" $4^{a}$ edição COVID-19- Guia Orientador para o enfrentamento da pandemia na Rede de Atenção à Saúde", [s.d.]), pois, em muitos casos, são expostos diretamente a pacientes infectados. Além disso, existem os fatores ambientais, como alto estresse ao atender pacientes em estado grave, condições de trabalho, às vezes, inadequada, e o receio de contaminar familiares (ORNELL et al., 2020; PAPPA et al., 2020; SILVA et al., 2021; TEIXEIRA et al., 2020).

Nesse contexto, este trabalho busca mostrar o impacto da imunização por meio das vacinas Corona Vac/Instituto Butantan e AstraZeneca/Fiocruz no grupo prioritário de profissionais da saúde, nos cinco municípios mais populosos do RS: Porto Alegre, Caxias do Sul, Canoas, Pelotas e Santa Maria.

\section{METODOLOGIA}

Esse trabalho foi desenvolvido a partir do acesso aos números de casos da COVID-19 no estado do RS por meio dos serviços de transparência pública, como o Painel Coronavírus RS(“Painel Coronavírus RS”, [s.d.]), painel síndrome respiratória aguda grave (SRAG) e base de vacinação("Painel Coronavírus RS", [s.d.]), além de dados obtidos diretamente das secretarias de saúde dos municípios estudados. Com base nas planilhas disponibilizadas, foram feitas intersecções de dados para selecionar informações relevantes como casos diagnosticados por semana epidemiológica, início do ciclo de vacinação, cidades, entre outros. Especificamente para os profissionais de saúde, foram identificadas as datas de diagnóstico positivo integralmente para o ano de 2020 e até 23 de junho de 2021, demarcado o final de 
EDUCAÇÃO, SAÚDE

ETECNOLOGIA

26 A 28 DE OUTUBRO DE 2021

vacinação da maioria deste grupo e observada a frequência de casos por semana epidemiológica.

Para análise, foram selecionados os cinco municípios mais populosos do RS sob condição de observar o efeito da vacinação nos profissionais de saúde, tanto pelo quantitativo de trabalhadores ativos, assim como por essas cidades serem centros de referência para o tratamento da COVID-19 em suas macrorregiões("Painel Coronavírus RS", [s.d.]). Os municípios selecionados foram Porto Alegre, Caxias do Sul, Canoas, Pelotas e Santa Maria.

Nesse cenário foram calculadas regressões não lineares, utilizando a biblioteca científica SciPy, para modelar o comportamento futuro das notificações a partir do ajuste de curva durante toda a pandemia até o momento da aplicação da primeira dose da vacina. O método resolve o modelo $y \sim f(x, \beta)$, onde y relaciona um vetor de variáveis independentes $x$, e suas variáveis dependentes observadas associadas, $y$. A função $f$ é não linear nos componentes do vetor de parâmetros $\beta$, mas de forma arbitrária. Destaca-se que as curvas foram geradas a partir de dados considerando: i) grupo sem vacinação; ii) grupo imunizado; e iii) média semanal acumulada.

\section{RESULTADOS E DISCUSSÕES}

A partir da planilha do Painel Coronavírus RS para notificações positivas para COVID-19("Painel Coronavírus RS”, [s.d.]), foi possível selecionar o conjunto de casos positivos para pacientes positivos cuja marcação "PROFISSIONAL_SAUDE" era anotada como "SIM".

No conjunto de pacientes profissionais de saúde foi possível segmentar os casos por município e agrupados por semana epidemiológica, conforme a data de confirmação da notificação. Da mesma forma, foi associada a data final de vacinação a partir de um segundo banco de dados, contendo todas as notificações 
de aplicação de vacina - incluindo o tipo da vacina, município e data da aplicação possibilitando identificar a semana epidemiológica de imunização da classe.

Destaca-se que a imunização dos profissionais da saúde, até 23 de junho de 2021, já atingiu primeira e segunda dose de vacinas nos respectivos municípios de acordo com os dados: Porto Alegre - $1^{\mathrm{a}}$ dose 126.795 e $2^{\mathrm{a}}$ dose 109.088, Caxias do Sul - $1^{\mathrm{a}}$ dose 20.708 e $2^{\mathrm{a}}$ dose 15.271 , Canoas - $1^{\mathrm{a}}$ dose 13.351 e $2^{\mathrm{a}}$ dose 11.042 , Pelotas - $1^{\mathrm{a}}$ dose 19.545 e $2^{\mathrm{a}}$ dose 14.361 , Santa Maria - $1^{\mathrm{a}}$ dose 12.903 e $2^{\mathrm{a}}$ dose

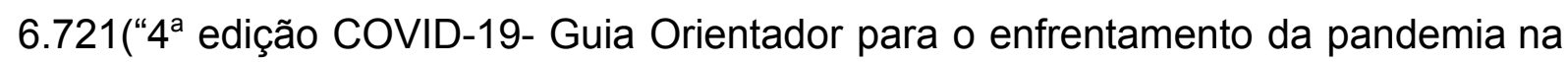
Rede de Atenção à Saúde", [s.d.]).

$\mathrm{Na}$ coleta de dados nos casos positivos para a COVID-19, foi possível identificar 3.500 listados em Porto Alegre, sendo 3.162 em 2020 e 573 em 2021 (Tabela 1). Em 2020 houveram dois picos de notificações na cidade, indicados pelas setas na coluna da esquerda na Figura 1. Observando o comportamento das notificações foi possível calcular a regressão sem vacinação (mostrada em vermelho na Figura 1), calculada pelo método curvefit, solução matemática para a determinação do polinômio que descreve os valores do intervalo de dados. A tendência para 2021 das notificações, sem vacinação, é crescente em todas as cidades conforme a curva de regressão em vermelho. 
Tabela 1- Total de casos positivos notificados no RS, nos municípios de Porto Alegre, Caxias do Sul, Canoas, Pelotas e Santa Maria para profissionais da saúde, e confirmados no ano de 2020 e 2021. Além disso, apresenta-se a previsão de casos com/sem a vacinação bem como a média semanal (total ano/semanas).

\begin{tabular}{|c|c|c|c|c|c|c|}
\hline Cidade & $\begin{array}{l}\text { Total de } \\
\text { casos- } \\
\text { profissiona } \\
\text { is da } \\
\text { saúde }\end{array}$ & $\begin{array}{l}\text { Casos } \\
\text { confirmad } \\
\text { os } 2020\end{array}$ & $\begin{array}{l}\text { Casos } \\
\text { confirmados } \\
2021\end{array}$ & $\begin{array}{l}\text { Previsto } \\
\text { s com } \\
\text { vacinaç } \\
\text { ão }\end{array}$ & $\begin{array}{l}\text { Previstos } \\
\text { sem } \\
\text { vacinaçã } \\
0\end{array}$ & $\begin{array}{l}\text { Média } \\
\text { semana } \\
\text { I (total } \\
\text { ano/se } \\
\text { manas) }\end{array}$ \\
\hline $\begin{array}{l}\text { Porto } \\
\text { Alegre }\end{array}$ & 3702 & 3162 & 573 & 2,53 & 60,25 & 29,52 \\
\hline $\begin{array}{l}\text { Caxias } \\
\text { do Sul }\end{array}$ & 2116 & 1598 & 518 & 0,75 & 53,31 & 19,18 \\
\hline Canoas & 1120 & 826 & 294 & 2,91 & 27,42 & 10,88 \\
\hline Pelotas & 1943 & 1384 & 559 & 1,89 & 48,01 & 20,55 \\
\hline $\begin{array}{l}\text { Santa } \\
\text { Maria }\end{array}$ & 1393 & 931 & 462 & 0,50 & 33,81 & 16,74 \\
\hline
\end{tabular}

Em 2021 os profissionais de saúde no RS iniciaram a imunização na segunda semana epidemiológica do ano, estendendo-se a campanha prioritária até a décima/ décima primeira semana, conforme mostrado na Figura 1, identificado com o asterisco $\left({ }^{*}\right)$ em cada cidade. Na coluna da direita na Figura 1, é possível observar que o ano de 2021 iniciou com um baixo número semanal de notificações, se comparado com os picos mostrados pelas setas na coluna da esquerda. O processo de vacinação possibilitou mudar o comportamento das curvas que definem as notificações, sendo então traçada uma nova regressão, agora levando em 
consideração a vacinação e gerando um novo traçado (em azul) que mostra a tendência do número semanal de notificações positivas em decréscimo.

Como comparativo, também foram calculadas as médias cumulativas a partir do início do ano de 2021, como forma de medir somente o comportamento da população do estudo durante o processo de vacinação. A média $\sum_{2}^{20} \operatorname{notif}(x) / n$, traçada em verde, após o marco de vacinação em todas as cidades modifica a tendência de crescimento mostrando estabilização ou comportamento decrescente.

Para o grupo de profissionais da saúde, na cidade de Canoas, foram registrados 826 casos em 2020 e 294 casos em 2021, enquanto em Caxias do Sul foram notificados, em 2020, 1.598 positivos e 518 em 2021. Em Santa Maria, em 2020, foram 931 casos e 462 positivos em 2021, já, na cidade de Pelotas, foram 1.384 casos positivos em 2020 e 559 em 2021, conforme os dados oficiais do RS [5] (Tabela 1). É importante salientar que, algumas secretarias municipais não atualizam imediatamente os dados de vacinação na plataforma estadual, podendo existir discrepâncias no número de vacinados no período. Foram analisados os diagnósticos positivos para COVID-19 de profissionais da saúde relatados, integralmente, no ano de 2020 até 23 de junho de 2021.

Figura 1 - Casos positivos notificados no RS, nos municípios de Porto Alegre (A), Caxias do Sul (B), Canoas (C), Pelotas (D) e Santa Maria (E) para profissionais da saúde. Na coluna da esquerda refere-se aos casos notificados no ano de 2020, com as setas indicando os picos de notificações atingidos no período e, na direita, para os casos notificados até 23 de junho de 2021 para cada município. As curvas pontilhadas em vermelho/azul indicam a regressão matemática dos dados sem/com imunização e a curva pontilhada verde indica a média semanal acumulada. 
EDUCAÇÃO, SAÚDE E TECNOLOGIA

26 A 28 DE OUTUBRO DE 2021

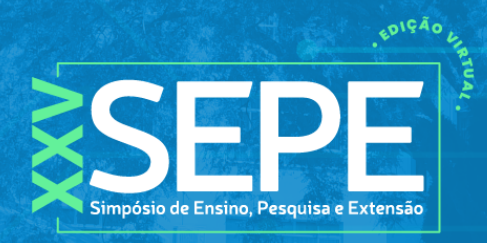

QUFN

(A)
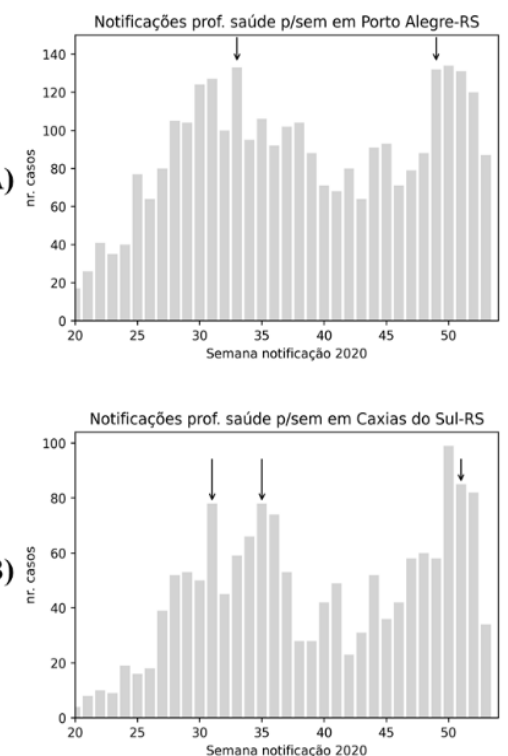

(C)

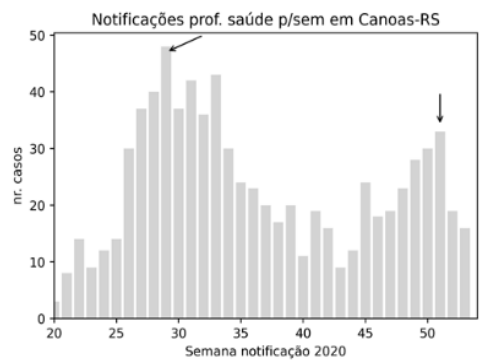

(D)

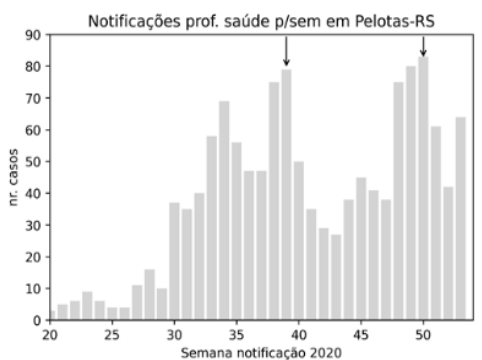

(E)

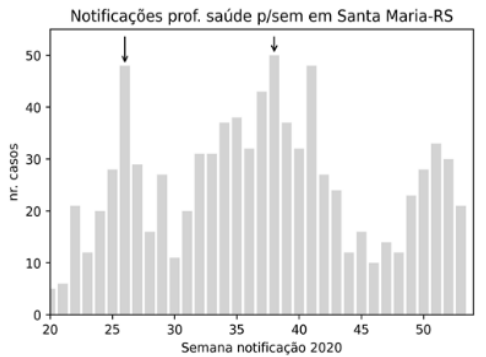

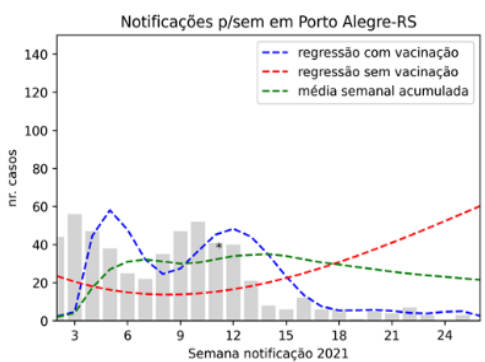
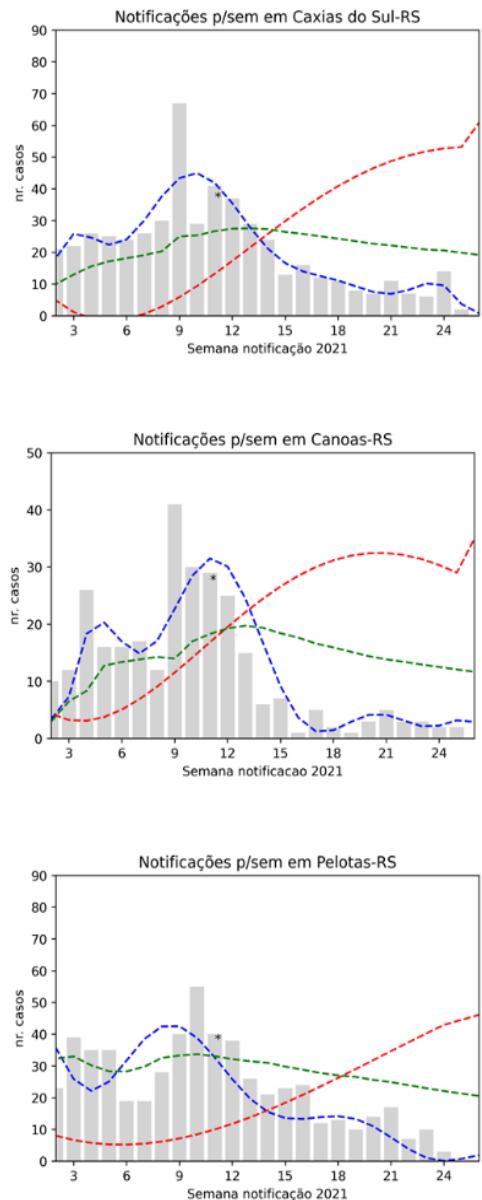

Notificações p/sem em Santa Maria-RS

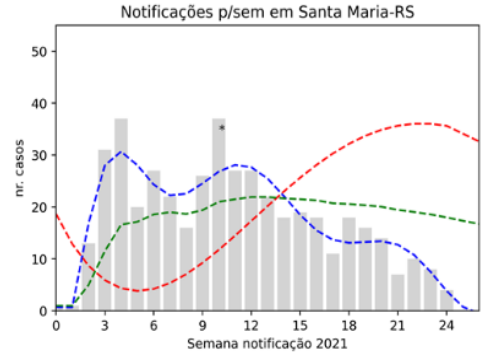

Fonte: Construção dos autores 
Conforme pode ser observado na Figura 1, as curvas de notificações positivas para a COVID-19, após a vacinação nos cinco municípios estudados, modificam seu comportamento quando comparado com a curva de regressão baseada no comportamento sem vacina (linha tracejada em vermelho). Enquanto nos profissionais de saúde, durante o ano de 2020, foram mantidas médias de notificações formando ondas nas semanas epidemiológicas, no ano de 2021, após a conclusão da vacinação, a curva que descreve os novos casos decresce, evitando a chamada "terceira onda" ao menos nas primeiras vinte semanas epidemiológicas de 2021. Nas cinco cidades pesquisadas não houveram notificações na vigésima sexta semana do ano, quando a média mensal em Porto Alegre era de 29 casos positivos, em Canoas de 10 casos, em Caxias do Sul esperados 19 em média, em Santa Maria 16 casos em média no ano de 2021 e em Pelotas a média esperada semanal calculada de 20 casos.

Um aspecto relevante é o perfil de trabalho dos profissionais da saúde, sendo que grande parte está na linha de frente do combate à COVID-19, altamente suscetível à contaminação ou até à reinfecção.

Estudos anteriores, sobre o impacto da vacinação pós-pandêmica contra a Influenza A (H1N1) (vírus da gripe) na cidade de Curitiba-PR, demonstram que a vacinação tende a diminuir a letalidade da infecção(GOLYNSKI, KELLY SANDRIN; MARQUES,CAMILA MORAES, 2015; VICTOR et al., 2014), consequentemente as taxas de hospitalização, havendo uma redução de casos a cada período analisado ${ }^{13,14}$. Dessa forma, é importante ressaltar que a vacinação é uma importante aliada no controle de doenças infecto-contagiosas, como no caso da COVID-19.

Portanto, os dados e modelos aqui apresentados reiteram a eficácia das vacinas, principalmente em grupos de profissionais ativos e em contato com o 
SarS-CoV-2, e a disponibilização equitativa em toda a sociedade é a única alternativa para superar a Pandemia da COVID-19.

\section{CONCLUSÃO}

Neste trabalho mostramos o impacto da imunização por meio das vacinas Corona Vac/Instituto Butantan e AstraZeneca/Fiocruz no grupo prioritário de profissionais da saúde, nos cinco municípios mais populosos do RS: Porto Alegre, Caxias do Sul, Canoas, Pelotas e Santa Maria. Os resultados, analisados até 23 de junho de 2021, mostram que ocorreu uma modificação do comportamento das curvas de notificações após o início da vacinação quando comparado com a curva de regressão baseada no comportamento sem vacina. Portanto, os modelos apresentados reiteram a eficácia das vacinas no enfrentamento da pandemia do COVID-19.

\section{AGRADECIMENTOS}

Projeto de pesquisa "Inteligência artificial em dados cadastrais e imagens na telemedicina em combate à COVID-19" - (Processo 88887.506897/2020-00) Edital nº 12/2020: CAPES-EPIDEMIAS - Programa Estratégico Emergencial de Prevenção e Combate a Surtos, Endemias, Epidemias e Pandemias.

\section{REFERÊNCIAS}

$4^{a}$ edição COVID-19- Guia Orientador para o enfrentamento da pandemia na Rede de Atenção à Saúde. , [s.d.]. Disponível em:

<https://www.conasems.org.br/wp-content/uploads/2021/04/Covid-19_guia_orientado r_4ed.pdf>

CARACILO CARVALHO BIVAR, G. et al. Covid-19, the anti-vaccine movement and immunization challenges in Brazil: A review. Scientia Medica, v. 31, n. 1, p. e39425, 18 fev. 2021.

GARCIA, L. P. Uso de máscara facial para limitar a transmissão da COVID-19.

Epidemiologia e Serviços de Saúde, v. 29, n. 2, maio 2020. 
GARCIA, L. P.; DUARTE, E. Intervenções não farmacológicas para o enfrentamento à epidemia da COVID-19 no Brasil. Epidemiologia e Serviços de Saúde, v. 29, n. 2, maio 2020.

GOLYNSKI, KELLY SANDRIN; MARQUES,CAMILA MORAES. AVALIAÇÃO EPIDEMIOLÓGICA DOS CASOS DE INFLUENZAA (H1N1) E O IMPACTO DA VACINAÇÃO EM INDIVÍDUOS QUE RESIDEM EM CURITIBA-PR. v. 2, p. 32-51, 15 jun. 2015.

LI, Q. et al. Early Transmission Dynamics in Wuhan, China, of Novel Coronavirus-Infected Pneumonia. New England Journal of Medicine, v. 382, n. 13, p. 1199-1207, 26 mar. 2020.

ORNELL, F. et al. The impact of the COVID-19 pandemic on the mental health of healthcare professionals. Cadernos de Saúde Pública, v. 36, n. 4, p. e00063520, 2020.

Painel Coronavírus RS. , [s.d.]. Disponível em: <https://ti.saude.rs.gov.br/covid19/>

PAPPA, S. et al. Prevalence of depression, anxiety, and insomnia among healthcare workers during the COVID-19 pandemic: A systematic review and meta-analysis.

Brain, Behavior, and Immunity, v. 88, p. 901-907, ago. 2020.

SILVA, D. F. O. et al. Prevalência de ansiedade em profissionais da saúde em tempos de COVID-19: revisão sistemática com metanálise. Ciência \& Saúde Coletiva, v. 26, n. 2, p. 693-710, fev. 2021.

TEIXEIRA, C. F. DE S. et al. A saúde dos profissionais de saúde no enfrentamento da pandemia de Covid-19. Ciência \& Saúde Coletiva, v. 25, n. 9, p. 3465-3474, set. 2020.

VICTOR, J. F. et al. Factors associated with vaccination against Influenza A (H1N1) in the elderly. Revista da Escola de Enfermagem da USP, v. 48, n. 1, p. 57-64, fev. 2014.

WU, F. et al. A new coronavirus associated with human respiratory disease in China. Nature, v. 579, n. 7798, p. 265-269, 12 mar. 2020.

ZHOU, P. et al. A pneumonia outbreak associated with a new coronavirus of probable bat origin. Nature, v. 579, n. 7798, p. 270-273, 12 mar. 2020. 